\title{
Does red ginseng ameliorate liver damage caused by obstructive jaundice?: an experimental study
}

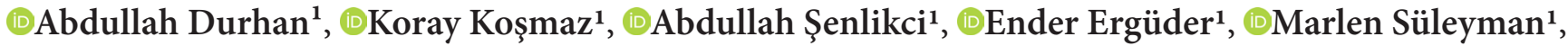

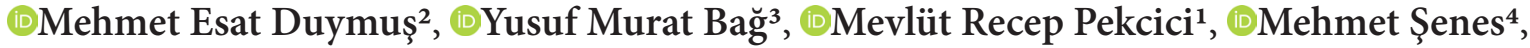 \\ ๑illknur Alkan Kuşabbi ${ }^{4}$, @Eylem Pınar Eser ${ }^{5}$, @Sema Hücümenoğlu ${ }^{5}$ \\ ${ }^{1}$ Ankara Training and Research Hospital, Department of General Surgery, Ankara, Turkey \\ ${ }^{2}$ Hatay State Hospital, Department of General Surgery, Hatay, Turkey \\ ${ }^{3}$ Van Training and Research Hospital, Department of General Surgery, Van, Turkey \\ ${ }^{4}$ Ankara Training and Research Hospital, Department of Biochemistry, Ankara, Turkey \\ ${ }^{5}$ Ankara Training and Research Hospital, Department of Pathology, Ankara, Turkey
}

Cite this article as: Durhan A, Koşmaz K, Şenlikci A, et al. Does red ginseng ameliorate liver damage caused by obstructive jaundice?: an experimental study. J Health Sci Med 2021; 4(2): 233-239.

\begin{abstract}
Aim: This experimental study aimed to evaluate the hepatoprotective effect on obstructive jaundice (OJ) of oral Red Ginseng (RG) extract, which is known to have anti-inflammatory and antioxidant properties.

Material and Method: The rats were randomly separated into 3 groups of 10 rats: The sham group, the control group, and the treatment group. In Group 1 (sham), the common bile duct (CBD) was identified but no ligation or transection was performed. In Group 2 (control), the CBD was identified and ligation and transection were performed, but no treatment was given. In Group 3 (RG group), CBD ligation and transection were performed, then RG extract was administered via an orogastric tube at a dose of $100 \mathrm{mg} / \mathrm{kg} /$ day for 10 days. After 10 days, blood samples were taken for biochemical analysis, and liver tissue samples for biochemical and histopathological analysis.

Results: Significantly higher serum albumin levels and lower serum alanine aminotransferase (ALT) and aspartate aminotransferase (AST) levels were determined in the RG group than in the control group $(\mathrm{p}=0.028, \mathrm{p}=0.001$ and $\mathrm{p}=0.034$, respectively). In the oxidative stress parameters, malondialdehyde (MDA) levels and catalase (CAT) levels were significantly different between the RG group and control group ( $\mathrm{p} \leq 0.001$ for each). Total sulfhydryl (T-SH) was not at a statistically significant level, although it was high and approached the value of the sham group $(\mathrm{p}=0.076)$. In the histopathological evaluation, the $\mathrm{RG}$ group had statistically significantly lower scores in all parameters compared to the control group $(\mathrm{p}<0.05)$.

Conclusion: The results of this study showed that RG has a strong hepatoprotective effect as a result of its anti-inflammatory and antioxidant properties.
\end{abstract}

Keywords: Red ginseng, obstructive jaundice, anti-inflammatory properties, antioxidant properties, hepatoprotective effects

\section{INTRODUCTION}

The immune response is the most important defence system that protects the human body against attacks from harmful micro-organisms, and toxic, chemical, and carcinogenic substances. One of the mechanisms of the immune system is the balance between the pro-inflammatory cytokines and anti-inflammatory cytokines secreted in the body. Inflammation is the disruption of this homeostasis in favor of pro-inflammatory cytokines, and this forms the basis of many diseases ranging from various autoimmune diseases to different types of cancer (1-3). Not only is the liver a vital human organ in terms of metabolic, synthetic, and detoxification functions, but it is also the body's antioxidant and inflammatory balance store. Despite advances in technology and imaging techniques, liver diseases are still diagnosed at a late stage (4). The first sign of liver diseases is usually jaundice. Obstructive jaundice $(\mathrm{OJ})$ may be due to intrahepatic diseases or generally due to diseases that cause extrahepatic biliary obstruction.

OJ may lead to liver-specific diseases that can progress to cholangitis, cirrhosis, and organ failure, and may result in many clinical sequelae if the treatment is not applied in a timely and adequate manner and bile flow 
is not provided. The clinical entities it may reveal are renal failure, encephalopathy, gastrointestinal system bleeding, coagulation disorders, malnutrition, and sepsis (5). Of these, sepsis is a very serious problem due to its consequences. An important consequence of obstructive liver disease in humans and experimental animals is hepatic inflammation, which causes tissue damage with the release of inflammatory cytokines and toxic free oxygen radicals (6). Oxidative stress and lipid peroxidation cause significant damage to the liver kupffer cells and other tissues, causing these cells to not work effectively in removing lipopolysaccharides and other toxins. In this context, antioxidant therapy is effective on an experimental basis. Although many studies have been conducted using various antioxidant and antiinflammatory agents to prevent or reverse oxidative stress, which has an important role in the pathogenesis of OJ, there is no commonly used drug in clinical practice (6-8).

Panax ginseng is one of the most widely used herbal remedies in Asia and Western countries. Ginseng is a slow-growing succulent perennial plant, the root of which is made up of ginsenoids and the most important sub-component is red ginseng (RG). RG has been used in traditional medicine for many years in far eastern countries such as China, Korea, and Japan, and there are studies of the use of RG in many fields of medicine such as liver, cardiovascular diseases, kidney diseases, autoimmune systemic diseases, various malignancy diseases and in areas such as strengthening the immune system (9-11). Studies have shown that RG reveals anti-inflammatory activities in inflammatory responses by regulating the activities of inflammatory signalling pathways (12). Recent studies have shown the antioxidant and anti-inflammatory effects of RG in reducing the toxic effects of hepatoxic drugs and agents (11). According to the literature, this is the first study to examine the effect of RG on liver damage due to $\mathrm{OJ}$.

The aim of this study was to evaluate the hepatoprotective effect of oral RG extract, which is known to have anti-inflammatory and antioxidant properties, on an experimental model of $\mathrm{OJ}$.

\section{MATERIAL AND METHOD}

The experimental procedures and technique of this study met the requirements of the National Guidelines for the Use and Care of Laboratory Animals and The Animal Ethics Committee approved this study (approval date: 26.11.2020, number: 0063: 640). All procedures were performed adhered to the ethical rules and principles.

\section{Animals and Experimental Surgical Procedure}

The study sample consisted of 30 female adult Wistar albino rats, each weighing $230 \pm 22 \mathrm{~g}$. The rats were kept in wire mesh cages at a permanent temperature of $21 \pm 2^{\circ}$ $\mathrm{C}$ with a 12-hour light / dark cycle. The animals were fed a standard lab food diet with drinking water ad libitum. Access to food was stopped 12 hours before anesthesia and to water 2 hours before anesthesia. The same team carried out the anesthesia and surgical intervention stages in sterile conditions. Before the interventions, the rats were anaesthesized with an intramuscular injection of $50 \mathrm{mg} /$ kg ketamine hydrochloride (Ketalar; Parke-Davis, Detroit, MI, USA) and $5 \mathrm{mg} / \mathrm{kg}$ Xylazine (Rompun; Bayer AG, Leverkusen, Germany). The rats were randomly separated into 3 groups of 10 rats: the sham group, the control group, and the treatment group. In Group 1 (sham), the common bile duct $(\mathrm{CBD})$ was identified and no ligation or transection was performed. In Group 2 (control), after the CBD was identified, dissected, and skeletonized, it was double tied using $5 / 0$ silk and cut between the sutures. No treatment was applied to this group. In Group 3 ( RG group ), following CBD ligation and transection, RG [ Korean 6-Year Root Red Ginseng Extract (Samsung, Korea) ] was administered via an orogastric tube at doses of $100 \mathrm{mg} / \mathrm{kg} /$ day for 10 days. After the specified procedures were completed, abdominal incisions were closed in two layers with $3 / 0$ silk sutures in all three groups. The rats were allowed to be fed after surgery. All the animals were euthanized after 10 days using an overdose of ketamine. Following laparotomy and sternotomy, samples were taken from liver tissue and blood to be analyzed biochemically, then liver tissue samples

\section{Biochemical Analysis}

Evaluation of the liver functions in the serum was carried out in the Biochemistry Department of Ankara Training and Research Hospital. Serum samples were kept at $-80^{\circ} \mathrm{C}$ until the day of analysis. Serum albumin, total protein, aspartate aminotransferase (AST), alanine aminotransferase (ALT), alkaline phosphatase (ALP), gamma-glutamyltransferase (GGT), total bilirubin, and direct bilirubin were measured by using a Roche Cobas 8000 biochemistry analyser.

The parameters of oxidative stress were evaluated in the Biochemistry Department of Ankara Training and Research Hospital. Liver tissues were kept at $-80^{\circ} \mathrm{C}$ until the day of analysis. The levels of malondialdehyde (MDA), total sulfhydryl (T-SH), and catalase (CAT) were determined. Tissues were homogenized in phosphate buffer for the MDA, T-SH, and CAT measurements.

The levels of MDA were measured using the fluorometric method, as described by Wasowicz et al. (13) The levels of MDA were shown as nmol/g protein. CAT levels were determined using spectrophotometric measurement as defined by Hadwan (14). The value of CAT activity was determined using the first-order reaction rate constant as $\mathrm{kU}$. CAT activity was stated as $\mathrm{kU} / \mathrm{g}$ protein. The T-SH measurement was performed according to the principle described by Taylan et al, who applied this method by adapting the Ellman reaction to the microplate method (15). The T-SH levels were stated as $\mu \mathrm{mol} / \mathrm{g}$ protein. 


\section{Histopathological Analysis}

The liver tissue samples were stored in containers containing $10 \%$ formol until the day of macroscopic examination. In the macroscopic examination, tissue specimens were cut into strips at $3 \mathrm{~mm}$ intervals and transferred to tissue tracking cassettes and embedded in paraffin blocks. Sections 4-microns in thickness were cut from the blocks and stained with Hematoxylin-Eosin and Masson Trichrome.

The pathological evaluation was performed by a single pathologist blinded to the groups. The sections prepared were examined under a light microscope at $\times 40, \times 100$, $\times 200$, and $\times 400$ magnifications. Histopathological examinations were evaluated using a modified histological activity index scoring system (HAI). The modified HAI scoring system consists of a total of five parameters: focal necrosis, portal inflammation, interphase hepatitis, confluent necrosis, and fibrosis (16).

In addition to HAI scoring, bile duct proliferation was evaluated. For the grading of bile duct proliferation, 5 consecutive areas were counted at $\mathrm{x} 100$ magnification and each proliferation was evaluated as 0 (none), 1 (mild), 2 (moderate), and 3 (severe). These numbers were then averaged and scored as $0=$ None, $1=$ Mild, $2=$ Moderate, $3=$ Severe.

\section{Statistical Analysis}

Data analysis was performed using IBM SPSS Statistics for Windows, version 25.0 software (IBM Corp., Armonk, N.Y., USA). The normality of the distribution of numerical variables was assessed using the Shapiro-Wilk test. Numerical variables were stated as median (minimummaximum) or mean \pm standard deviation (SD) values and were compared using the Kruskal-Wallis test followed by the Tamhane T2 post-hoc test or the one-way ANOVA test followed by Tukey's HSD post hoc test, as appropriate. A value of $\mathrm{p}<0.05$ was considered statistically significant.

\section{RESULTS}

Two rats in the control group died in the postoperative period and no other complication was observed during the study period.

\section{Liver function tests}

With the exception of total protein levels, all the other parameters differed significantly between the groups. Significantly lower serum albumin levels and higher levels of serum total protein, ALP, GGT, ALT, AST, total bilirubin, and direct bilirubin were determined in the control group compared to the sham group. With the exception of the ALT levels, these results were similar to the comparison of the RG group and the sham group.

Statistically significantly higher serum albumin levels and lower serum ALT and AST levels were determined in the RG group compared to the control group ( $\mathrm{p}=0.028$, $\mathrm{p}=0.001$, and $\mathrm{p}=0.034$ respectively). Although there was an improvement in ALP and GGT enzymes in the RG group compared to the control group, the difference was not statistically significant $(\mathrm{p}=0.139, \mathrm{p}=0.188$ respectively). The serum biochemical parameters of liver function are shown in Table 1.

\section{Oxidative stress parameters}

The MDA, CAT, and T-SH levels in liver tissue are presented in Table 2. The CAT and T-SH levels were significantly higher and MDA levels were significantly lower in the sham group compared to the control group and no significant difference was observed between the RG group and the sham group in these parameters. In the comparisons of the RG group and the control group, the MDA levels and CAT levels were significantly different $(\mathrm{p}=<0.001$ for each). T-SH was higher in the RG group than in the control group and approached the value of the sham group, but the difference was not statistically significant $(\mathrm{p}=0.076)$.

\begin{tabular}{|c|c|c|c|c|c|c|c|}
\hline & $\begin{array}{l}\text { Sham group } \\
\text { (Group 1) }\end{array}$ & $\begin{array}{l}\text { Control group } \\
\text { (Group 2) }\end{array}$ & $\begin{array}{l}\text { RG group } \\
\text { (Group 3) }\end{array}$ & $p$ value & $\underset{(1-2)}{p \text { value }}$ & $\underset{(1-3)}{p \text { value }}$ & $\underset{(2-3)}{p \text { value }}$ \\
\hline Albumin (g/L) & $37.22 \pm 2.19$ & $28.05 \pm 3.41$ & $31.68 \pm 2.74$ & $<0.001$ & $<0.001$ & $<0.001$ & 0.028 \\
\hline Total protein $(\mathrm{g} / \mathrm{L})$ & $57.69 \pm 3.90$ & $57.80 \pm 6.01$ & $58.91 \pm 2.48$ & 0.782 & 0.998 & 0.796 & 0.845 \\
\hline $\operatorname{ALP}(\mathrm{IU} / \mathrm{L})$ & $\begin{array}{c}95.5 \\
(68-162)\end{array}$ & $\begin{array}{c}435.5 \\
(219-850)\end{array}$ & $\begin{array}{c}275 \\
(209-399)\end{array}$ & $<0.001$ & 0.004 & $<0.001$ & 0.139 \\
\hline GGT (IU/L) & $\begin{array}{c}0 \\
(0-1)\end{array}$ & $\begin{array}{c}33 \\
(8-46)\end{array}$ & $\begin{array}{c}19 \\
(14-38)\end{array}$ & $<0.001$ & $<0.001$ & $<0.001$ & 0.188 \\
\hline ALT (IU/L) & $\begin{array}{c}67 \\
(51-100)\end{array}$ & $\begin{array}{c}148.5 \\
(101-189)\end{array}$ & $\begin{array}{c}87.5 \\
(41-113)\end{array}$ & $<0.001$ & $<0.001$ & 0.180 & 0.001 \\
\hline AST (IU/L) & $\begin{array}{c}237 \\
(175-288)\end{array}$ & $\begin{array}{c}477 \\
(369-829)\end{array}$ & $\begin{array}{c}336 \\
(211-417)\end{array}$ & $<0.001$ & 0.005 & 0.004 & 0.034 \\
\hline $\begin{array}{l}\text { Total bilirubin } \\
(\mathrm{mg} / \mathrm{dl})\end{array}$ & $\begin{array}{c}0.05 \\
(0.03-0.07)\end{array}$ & $\begin{array}{c}10.18 \\
(9.43-16.70)\end{array}$ & $\begin{array}{c}10.03 \\
(8.00-13.28)\end{array}$ & $<0.001$ & $<0.001$ & $<0.001$ & 0.820 \\
\hline $\begin{array}{l}\text { Direct bilirubin } \\
\text { (mg/dl) }\end{array}$ & $\begin{array}{c}0.02 \\
(0.02-0.04)\end{array}$ & $\begin{array}{c}9.80 \\
(8.54-16.00)\end{array}$ & $\begin{array}{c}9.68 \\
(6.88-13.00)\end{array}$ & $<0.001$ & $<0.001$ & $<0.001$ & 0.751 \\
\hline
\end{tabular}

RG: Red ginseng, ALP: Alkaline phosphatase, GGT: Gamma glutamyl transferase, ALT: Alanine aminotransferase, AST: Aspartate aminotransferase, Values shown in bold are statistically significant $(\mathrm{p}<0.05)$. 


\begin{tabular}{|lccccccc|}
\hline Table 2. Results and comparisons of malondialdehyde, catalase, and total sulfhydryl levels in liver tissue & & \\
& $\begin{array}{c}\text { Sham group } \\
\text { (Group 1) }\end{array}$ & $\begin{array}{c}\text { Control group } \\
\text { (Group 2) }\end{array}$ & $\begin{array}{c}\text { RG group } \\
\text { (Group 3) }\end{array}$ & $\begin{array}{c}\text { p } \\
\text { value }\end{array}$ & $\begin{array}{c}\text { P value } \\
(\mathbf{1 - 2})\end{array}$ & $\begin{array}{c}\text { P value } \\
(\mathbf{1 - 3})\end{array}$ & $\begin{array}{c}\text { P value } \\
(\mathbf{2}-3)\end{array}$ \\
\hline MDA (nmol/g protein) & $65.42 \pm 17.45$ & $102.13 \pm 5.58$ & $76.28 \pm 6.40$ & $<0.001$ & $<0.001$ & 0.109 & $<0.001$ \\
Catalase $(\mathrm{kU} / \mathrm{g}$ protein) & $44.64 \pm 5.66$ & $27.12 \pm 6.41$ & $42.91 \pm 6.47$ & $<0.001$ & $<0.001$ & 0.807 & $<0.001$ \\
Total SH $\mu \mathrm{mol} / \mathrm{g}$ protein & $167.51 \pm 23.84$ & $129.66 \pm 16.16$ & $151.23 \pm 18.09$ & $<0.001$ & 0.001 & 0.180 & 0.076 \\
\hline RG: Red ginseng, MDA: Malondialdehyde, SH: Sulfhydryl, Values shown in bold are statistically significant $(\mathrm{p}<0.05)$ & & & \\
\hline
\end{tabular}

\section{Histopathological Results}

The scores of the histopathological changes in the liver tissue are summarized in Table 3. The sham group had the lowest scores as expected and all parameters were significantly lower compared to the control group. The RG group had significantly lower scores for all parameters compared to the control group. In the comparison of the RG and sham groups, statistically different changes were determined in four of six parameters (Figure 1).

\section{DISCUSSION}

Benign and malignant diseases are among the causes of OJ. Biliary obstruction is known to cause hepatocellular damage, primarily, as a result of blocking the passage of bile into the intestine, high concentrations of bile acid accumulate in intrahepatic sinusoids, hepatocytes, and blood, and this triggers oxidative stress and inflammatory response (6). Inflammatory cell infiltration, accumulation of hydrophobic bile acids, endotoxemia, changes in mitochondrial permeability transition, and deleterious effects of oxygen free radicals are possible factors responsible for cholestatic liver damage (17, 18). These radicals damage the cell membrane as well as intracellular structures. Decreased absorption from the intestines causes the plasma levels of antioxidants to decrease and thus increases oxidative damage. Oxidative damage and lipid peroxidation exacerbate liver damage in OJ (19). Although the mechanism of liver damage due to $\mathrm{OJ}$ is complex and multifactorial, the two main factors affecting the degree of liver damage are oxidative stress and inflammation. In the literature, many experimental
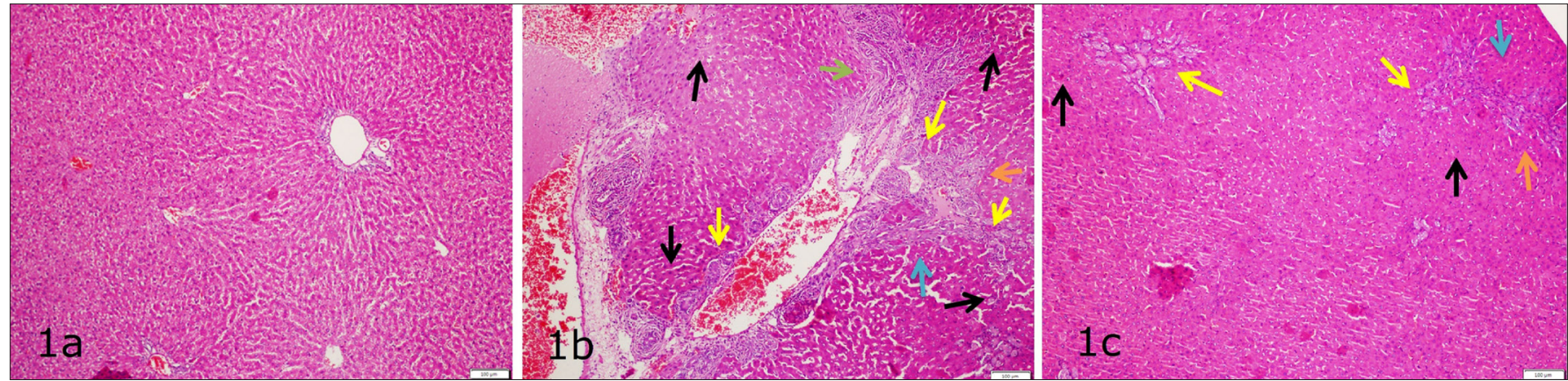

Figure 1. Histopathological findings: 1a: Normal microanatomy of the liver was preserved. There is no portal inflammation, fibrosis and significant bile duct proliferation in the portal area. (H\&E x100; Sham group). 1b: Fibrous septa / bridging and nodules caused by severe biliary duct proliferation (yellow arrow). Nodules consist of fibrous (green arrow) and inflammatory bridges (blue arrow) in some areas. Focal necrosis foci and prominent necrotic keratinocytes (black arrow) are observed in hepatocytes. There are signs of severe interphase hepatitis (orange arrow) in the portal areas (H\&E x100; Control group). 1c: Sparse focal necrosis and necrotic keratinocyte foci (black arrow). Mild inflammation and fibrosis (blue arrow) and focal interphase hepatitis findings (orange arrow) are seen in one of the two Portal areas. Mild bile ductus proliferation (yellow arrow) is observed in both portal areas. (H\&E x100; RG group).

\begin{tabular}{|c|c|c|c|c|c|c|c|}
\hline & $\begin{array}{c}\text { Sham group } \\
\text { (Group 1) }\end{array}$ & $\begin{array}{l}\text { Control group } \\
\text { (Group 2) }\end{array}$ & $\begin{array}{l}\text { RG group } \\
\text { (Group 3) }\end{array}$ & $\underset{\text { value }}{\mathbf{p}}$ & $\underset{(1-2)}{p \text { value }}$ & $\begin{array}{c}p \text { value } \\
(1-3)\end{array}$ & $\begin{array}{c}p \text { value } \\
(2-3)\end{array}$ \\
\hline Bile duct proliferation & $\begin{array}{c}0 \\
(0-1)\end{array}$ & $\begin{array}{c}3 \\
(2-3)\end{array}$ & $\begin{array}{c}2 \\
(1-3)\end{array}$ & $<0.001$ & $<0.001$ & $<0.001$ & 0.001 \\
\hline Focal necrosis & $\begin{array}{c}0 \\
(0-1)\end{array}$ & $\begin{array}{c}2 \\
(1-3)\end{array}$ & $\begin{array}{c}1 \\
(0-2)\end{array}$ & 0.001 & 0.001 & 0.309 & 0.024 \\
\hline Portal inflammation & $\begin{array}{c}1 \\
(0-1)\end{array}$ & $\begin{array}{c}3 \\
(2-4)\end{array}$ & $\begin{array}{c}1 \\
(1-2)\end{array}$ & $<0.001$ & $<0.001$ & 0.008 & 0.001 \\
\hline Interface hepatitis & $\begin{array}{c}0 \\
(0-1)\end{array}$ & $\begin{array}{c}3 \\
(3-4)\end{array}$ & $\begin{array}{c}1.5 \\
(0-2)\end{array}$ & $<0.001$ & $<0.001$ & 0.025 & $<0.001$ \\
\hline Confluent necrosis & $\begin{array}{c}0.5 \\
(0-2)\end{array}$ & $\begin{array}{c}3 \\
(2-3)\end{array}$ & $\begin{array}{c}1 \\
(0-2)\end{array}$ & $<0.001$ & $<0.001$ & 0.252 & $<0.001$ \\
\hline Fibrosis & $\begin{array}{c}1 \\
(0-1)\end{array}$ & $\begin{array}{c}3 \\
(2-4)\end{array}$ & $\begin{array}{c}2 \\
(1-2)\end{array}$ & $<0.001$ & $<0.001$ & 0.002 & $<0.001$ \\
\hline
\end{tabular}


studies have been conducted with various drugs with antioxidant and anti-inflammatory properties to treat the damage caused by OJ. In an experimental study of OJ, Unal et al reported that Calcium Dobecylate significantly reduced the development of inflammation and fibrosis in the liver, and that serum and tissue enzyme activity decreased biochemically and oxidative stress significantly (20). Moran et al. demonstrated that nitric oxide (NO) plays a crucial role in inducing liver damage in rats with jaundice and that erythropoietin reduces oxidative damage both by decreasing NO levels and increasing or possibly preserving reduced glutathione levels (6). Padillo et al. reported that melatonin reduced liver damage in an experimental cholestasis study (17). In the literature, there are studies that have shown that OJ causes damage to distant organs as well as having a toxic effect on the liver. In one of these, it was stated that there is a relationship between high lipid peroxidation and damage to the heart, brain, and kidney in experimental OJ (21). In another experimental cholestasis study, Dilektasli et al. found brain damage to be irreversible if the duration of bile duct obstruction was $>7$ days (22). A notable study from the experimental OJ studies was about the method of creating obstruction in the common bile duct. Yunfu et al. compared the methods of CBD ligation and transection with CBD ligation only and showed that the total bilirubin level returned to normal on day 21 only in the rats that were ligated, and therefore recommended the CBD ligation and transection method for experimental cholestasis studies (23). In the current study, OJ was created by the CBD ligation and transection method.

Ginseng, a plant belonging to the Panax genus, belongs to the Araliaceae family and has been used as a popular herbal medicine for thousands of years, especially in Far East Asia. There are 13 plants related to the Panax genus, the most commonly used of which is Panax ginseng (24). RG has traditionally been shown in studies to be more effective than white or fresh ginseng (25). Most of the biological activities of ginseng are due to its main constituent, ginsenosides. Also known as steroidlike saponins, ginsenosides are unique to the ginseng types. There are more than 100 ginsenosides expressed by $\mathrm{Rx}$ (26). Various studies have been conducted to understand the pharmacological mechanisms of ginseng and ginsenosides in diabetes mellitus, cardiovascular disease, stress, neurodegenerative diseases, various types of cancer, and immuno-stimulation $(9,27,28)$.

Inflammation is one of the natural immune mechanisms. While the dynamic balance between proinflammatory cytokines and anti-inflammatory cytokines modulates the state of inflammation, disruption of the balance in favour of pro-inflammatory cytokines causes inflammation-related diseases such as diabetes, cancer, cardiovascular disease, and neurological diseases. Considering the mechanism of action of ginsenosides, its anti-inflammatory effects have been proven by purified ginsenosides. Pro-inflammatory cytokine expressions (TNF-alpha, IL-1, and IL-6) have been shown to inhibit iNOS and COX-2 enzyme expressions while inducing M1 and M2 polarization of macrophages or microglia, which in turn has been found to be the anti-inflammatory mechanism of ginsenosides $(12,29,30)$. An important consequence of obstructive liver disease in humans and experimental animals is hepatic inflammation, which causes tissue damage with the release of inflammatory cytokines and toxic free oxygen radicals. Oxidative stress and lipid peroxidation cause significant damage to the liver kupffer cells and other tissues, causing these cells to not work effectively in removing lipopolysaccharides and other toxins. In this context, antioxidant therapy is effective on an experimental basis (6). In current study, liver damage caused by obstructive jaundice in the control group and RG group caused an increase in liver function tests and oxidative stress parameters in both groups, but these values healed significantly in the RG group compared to the control group. We think that the antiinflammatory and antioxidant effects of ginsenosides are very important in this healing process.

There are many studies in literature which have examined the hepatoprotective effect besides the anti-inflammatory effect of RG. Karakus et al. stated in an experimental study that serum biochemical liver function tests, which increased in liver damage due to carbon tetrachloride (CCl4) toxicity, improved significantly in rats receiving $R G$ treatment (31). In another experimental study by Hong et al., 2 months of oral RG treatment in rats with nonalcoholic fatty liver was shown to inhibit steatohepatitis, improve high lipid profile values, and stimulate natural clay cell activity (32). RG administration has also been shown to provide a significant improvement in liver function tests in patients with chronic hepatitis $\mathrm{C}$, lower viral titers, and lower the level of tumor marker alpha-fetoprotein (AFP) by showing potent therapeutic effects against liver cancer (33). In a recent experimental study, it was stated that serum liver function tests and oxidative parameters improved in the group treated with RG in rats given cyclophosphamide, which has hepatotoxic side effects, and its hepatoprotective effect was emphasized (11).

In this study, the histopathological evaluation of liver tissue samples and biochemically oxidative and antioxidant parameters of blood samples were examined. RG was found to significantly reduce inflammation, necrosis, and fibrosis in the histopathological evaluation. It was also observed that oxidative stress parameters and liver function tests improved significantly in the RG group compared to the control group. 


\section{CONCLUSION}

In conclusion, the results of this study showed that RG had a strong hepatoprotective effect as a result of its antiinflammatory and antioxidant properties. To the best of our knowledge, this is the first study in literature to have investigated the effect of RG on experimental hepatic OJ. Although promising beneficial hepatoprotective effects of RG have been demonstrated, there is a need for further clinical studies to apply these positive results in clinical settings.

\section{ETHICAL DECLARATIONS}

Ethics Committee Approval: The experimental procedures and technique of this study met the requirements of the National Guidelines for the Use and Care of Laboratory Animals and Ankara Training and Research Hospital The Animal Ethics Committee approved this study (approval date: 26.11.2020, number: 0063: 640). All procedures were performed adhered to the ethical rules and principles.

Referee Evaluation Process: Externally peer-reviewed.

Conflict of Interest Statement: The authors have no conflicts of interest to declare.

Financial Disclosure: The authors declared that this study has received no financial support.

Author Contributions: All of the authors declare that they have all participated in the design, execution, and analysis of the paper, and that they have approved the final version.

\section{REFERENCES}

1. González-Chávez A, Elizondo-Argueta S, Gutiérrez-Reyes G, León-Pedroza JI. Pathophysiological implications between chronic inflammation and the development of diabetes and obesity. Cir Cir 2011; 79: 209-16.

2. Tracy RP. Emerging relationships of inflammation, cardiovascular disease and chronic diseases of aging. Int J Obes Relat Metab Disord 2003; 27: 29-34.

3. Allavena P, Germano G, Marchesi F, Mantovani A. Chemokines in cancer related inflammation. Exp Cell Res 2011; 317: 664-73.

4. Baeck C, Tacke F. Balance of inflammatory pathways and interplay of immune cells in the liver during homeostasis and injury. EXCLI J 2014; 13: 67-81.

5. Scott-Conner CE, Grogan JB. The pathophysiology of biliary obstruction and its effect on phagocytic and immune function. J Surg Res 1994; 57: 316-36.

6. Moran M, Oruc MT, Ozmen MM, et al. Effect of erythropoietin on oxidative stress and liver injury in experimental obstructive jaundice. Eur Surg Res 2009; 43: 228-34.

7. KoŞmaz K, Durhan A, SÜleyman M, et al. The Effect of Ankaferd Blood Stopper on Liver Damage in Experimental Obstructive Jaundice. Turk J Med Sci 2020. doi:10.3906/sag-2007-298.

8. Kismet K, Sabuncuoglu MZ, Kilicoglu SS, et al. Effect of propolis on oxidative stress and histomorphology of liver tissue in experimental obstructive jaundice. Eur Surg Res 2008; 41: 231-7.
9. Ahuja A, Kim JH, Kim JH, Yi YS, Cho JY. Functional role of ginseng-derived compounds in cancer. J Ginseng Res 2018; 42: 248-54.

10. Huu Tung N, Uto T, Morinaga O, Kim YH, Shoyama Y. Pharmacological effects of ginseng on liver functions and diseases: a minireview. Evid Based Complement Alternat Med 2012; 2012: 173297.

11. Abdelfattah-Hassan A, Shalaby SI, Khater SI, El-Shetry ES, Abd El Fadil H, Elsayed SA. Panax ginseng is superior to vitamin E as a hepatoprotector against cyclophosphamide-induced liver damage. Complement Ther Med 2019; 46: 95-102.

12. Kim JH, Yi YS, Kim MY, Cho JY. Role of ginsenosides, the main active components of Panax ginseng, in inflammatory responses and diseases. J Ginseng Res 2017; 41: 435-43.

13. Wasowicz W, Nève J, Peretz A. Optimized steps in fluorometric determination of thiobarbituric acid-reactive substances in serum: importance of extraction $\mathrm{pH}$ and influence of sample preservation and storage. Clin Chem 1993; 39: 2522-6.

14. Hadwan MH. Simple spectrophotometric assay for measuring catalase activity in biological tissues. BMC Biochem 2018; 19: 7.

15. Taylan E, resmi h. The Analytical Performance of a Microplate Method for Total Sulfhydryl Measurement in Biological Samples. Turk J Biochem 2010; 35: 275-8.

16. Ishak KG. Pathologic features of chronic hepatitis. A review and update. Am J Clin Pathol 2000; 113: 40-55.

17. Padillo FJ, Cruz A, Navarrete C, et al. Melatonin prevents oxidative stress and hepatocyte cell death induced by experimental cholestasis. Free Radic Res 2004; 38: 697-704.

18. Chuang JH, Chang NK, Huang CC, et al. Biliary intervention augments chemotactic reaction and aggravates cholestatic liver injury in rats. J Surg Res 2004; 120: 210-8.

19. Cantürk NZ, Canturk Z, Utkan NZ, et al. Cytoprotective effects of alpha tocopherol against liver injury induced by extrahepatic biliary obstruction. East Afr Med J 1998; 75: 77-80.

20. Unal Y, Tuncal S, Kosmaz K, et al. The Effect of Calcium Dobesilate on Liver Damage in Experimental Obstructive Jaundice. J Invest Surg 2019; 32: 238-44.

21.Ljubuncic P, Tanne Z, Bomzon A. Evidence of a systemic phenomenon for oxidative stress in cholestatic liver disease. Gut 2000; 47: 710-6.

22.Dilektasli E, Ozmen MM, Gundogdu E, Dizen H, Besler HT, Ozogul C. The effects of obstructive jaundice on the brain: An experimental study. Asian J Surg 2016; 39: 155-63.

23. Lv Y, Yue J, Gong X, et al. Spontaneous remission of obstructive jaundice in rats: Selection of experimental models. Exp Ther Med 2018; 15: 5295-301.

24. Gillis CN. Panax ginseng pharmacology: a nitric oxide link? Biochem Pharmacol 1997; 54: 1-8.

25.Xu XF, Gao Y, Xu SY, et al. Remarkable impact of steam temperature on ginsenosides transformation from fresh ginseng to red ginseng. J Ginseng Res 2018; 42: 277-87.

26. Chang-Xiao L, Pei-Gen X. Recent advances on ginseng research in China. J Ethnopharmacol 1992; 36: 27-38.

27. Hasegawa H. Proof of the mysterious efficacy of ginseng: basic and clinical trials: metabolic activation of ginsenoside: deglycosylation by intestinal bacteria and esterification with fatty acid. J Pharmacol Sci 2004; 95: 153-7.

28. Kim KH, Lee D, Lee HL, Kim CE, Jung K, Kang KS. Beneficial effects of Panax ginseng for the treatment and prevention of neurodegenerative diseases: past findings and future directions. J Ginseng Res 2018; 42: 239-47.

29.Lü JM, Yao Q, Chen C. Ginseng compounds: an update on their molecular mechanisms and medical applications. Curr Vasc Pharmacol 2009; 7: 293-302. 
30.Im DS. Pro-Resolving Effect of Ginsenosides as an AntiInflammatory Mechanism of Panax ginseng. Biomolecules 2020; 10: 444 .

31. Karakus E, Karadeniz A, Simsek N, et al. Protective effect of Panax ginseng against serum biochemical changes and apoptosis in liver of rats treated with carbon tetrachloride (CCl4). J Hazard Mater 2011; 195: 208-13.

32. Hong SH, Suk KT, Choi SH, et al. Anti-oxidant and natural killer cell activity of Korean red ginseng (Panax ginseng) and urushiol (Rhus vernicifera Stokes) on non-alcoholic fatty liver disease of rat. Food Chem Toxicol 2013; 55: 586-91.

33. Abdel-Wahhab PM, Gamil K, Elkady A, el-nekeety A, Naguib K. Therapeutic Effects of Korean Red Ginseng Extract in Egyptian Patients with Chronic Liver Diseases. J Ginseng Res 2011; 35: 6979. 